\title{
IrSD Thrombosis
}

National Cancer Institute

\section{Source}

National Cancer Institute. irSD Thrombosis. NCI Thesaurus. Code C147913.

Any of a) no change or small changes that do not meet the criteria for irPR or irPD,

taking as reference the smallest sum diameters while on study, b) increase in the volume of non-enhancing thrombosis, c) new bland non-enhancing thrombosis. 